\title{
Long-term effects on luminal and mucosal microbiota and commonly acquired taxa in faecal microbiota transplantation for recurrent Clostridium difficile infection
}

\author{
Jonna Jalanka', Eero Mattila², Hanne Jouhten ${ }^{1}$, Jorn Hartman ${ }^{3}$, Willem M. de Vos ${ }^{1,3}$, Perttu Arkkila ${ }^{4}$
} and Reetta Satokari, ${ }^{1,5^{*}}$

\begin{abstract}
Background: Faecal microbiota transplantation (FMT) is an effective treatment for recurrent Clostridium difficile infection ( $\mathrm{rCDI}$ ). It restores the disrupted intestinal microbiota and subsequently suppresses $C$. difficile. The long-term stability of the intestinal microbiota and the recovery of mucosal microbiota, both of which have not been previously studied, are assessed herein. Further, the specific bacteria behind the treatment efficacy are also investigated.

Methods: We performed a high-throughput microbiota profiling using a phylogenetic microarray analysis of 131 faecal and mucosal samples from $14 \mathrm{rCDI}$ patients pre- and post-FMT during a 1-year follow-up and 23 samples from the three universal donors over the same period.

Results: The FMT treatment was successful in all patients. FMT reverted the patients' bacterial community to become dominated by Clostridium clusters IV and XIVa, the major anaerobic bacterial groups of the healthy gut. In the mucosa, the amount of facultative anaerobes decreased, whereas Bacteroidetes increased. Post-FMT, the patients' microbiota profiles were more similar to their own donors than what is generally observed for unrelated subjects and this striking similarity was retained throughout the 1-year follow-up. Furthermore, the universal donor approach allowed us to identify bacteria commonly established in all CDI patients and revealed a commonly acquired core microbiota consisting of 24 bacterial taxa.

Conclusions: FMT induces profound microbiota changes, therefore explaining the high clinical efficacy for rCDI. The identification of commonly acquired bacteria could lead to effective bacteriotherapeutic formulations. FMT can affect microbiota in the long-term and offers a means to modify it relatively permanently for the treatment of microbiotaassociated diseases.
\end{abstract}

Keywords: Bacteriotherapy, Microbiome, Universal donor, Mucosal bacteria

\section{Background}

The incidence of Clostridium difficile infections (CDI) has increased, with up to $50 \%$ of patients developing recurrent infections $[1,2]$. The bacterium is the main etiological agent of antibiotic-associated diarrhoea, causing a major burden to the healthcare system [3-5]. Diverse

\footnotetext{
* Correspondence: reetta.satokari@helsinki.fi

${ }^{1}$ Immunobiology Research Program and Department of Bacteriology and Immunology, Faculty of Medicine, University of Helsinki, Helsinki, Finland ${ }^{5}$ PO Box 21, Haartmaninkatu 3, 00290 Helsinki, Finland

Full list of author information is available at the end of the article
}

intestinal microbiota provides colonisation resistance against pathogens and perturbations to the normal microbiota introduced by an antibiotic treatment is a key step in CDI pathogenesis [5]. Traditionally, CDI is treated with metronidazole or vancomycin and, more recently, with fidaxomicin and rifaximin $[2,6,7]$. These antimicrobials devastate the intestinal microbiota even further. If $C$. difficile spores persist after antibiotic treatment they can germinate and proliferate in the absence of suppressing microbiota and, as a consequence, the patient may enter a vicious cycle of recurrent CDI (rCDI) 
infections [8]. Further, the emerging antibiotic-resistant variants of $C$. difficile call for alternative treatment options [9].

Faecal microbiota transplantation (FMT) is highly effective in treating rCDI [10-13]. FMT from a healthy, pre-screened donor is placed into the patient's duodenum, cecum or rectum where it restores the diversity and composition of the disrupted microbiota and subsequently suppresses $C$. difficile [9, 11-18]. Emerging evidence suggests that FMT also restores secondary bile acid metabolism, which is impaired in rCDI and possibly has a role in disease development $[19,20]$. Several studies have followed the short-term stability of the transplanted microbiota and constituted that, overall, FMTinduced changes tend to persist over time $[14,16,17]$. Further, FMT's long-term clinical efficacy and safety have been demonstrated $[10,11,21]$. However, the longterm effects of FMT on microbiota have not been previously addressed, with prior work focusing on the effects on faecal microbiota rather than on the distinct ecosystem of mucosa.

Understanding the mechanistic basis of FMT treatment and the minimum microbial components necessary for a successful outcome are vital. Preliminary studies have been conducted, with evidence from a rCDI mouse model suggesting that a mixture of intestinal bacteria could be used instead of faecal material [22]. More recently, bacterial mixtures comprising over 30 strains were shown to resolve rCDI in two patients [23]. These results suggest that an effective treatment of CDI based on defined mixtures of bacteria might be feasible in the near future.

In this study, we aimed to build on the existing knowledge by concentrating on the long-term effects of FMT on the faecal microbiota as well as characterising rectal mucosal microbiota pre- and post-treatment. We used a universal donor approach, where several patients received their transplant from the same donor. This facilitated a controlled analysis of FMT-induced microbiota changes and the identification of key bacterial taxa that are commonly established in the gut of CDI patients. Thereby, we aimed to investigate the possibility of a commonly acquired core microbiota underlying the efficacy of the FMT treatment and which could be used as a basis for the design of bacteriotherapeutic formulations.

\section{Methods}

\section{Patients}

The intestinal microbiota of $14 \mathrm{rCDI}$ patients treated with FMT was analysed (Table 1; see the Additional file 1: Table S1 for detailed patient information and Fig. 1 and Additional file 1: Figure S1 for sample collection). All patients had laboratory-confirmed rCDI despite
Table 1 Patient demographics

\begin{tabular}{llllll}
\hline Patient no. & Age, years & Sex & Donor & Faecal material & Outcome \\
\hline P1 & 63 & Male & D2 & Frozen & Resolution \\
P2 & 45 & Male & D2 & Frozen & Resolution \\
P3 & 88 & Female & D2 & Frozen & Resolution \\
P4 & 82 & Female & D2 & Frozen & Resolution \\
P5 & 81 & Female & D2 & Frozen & Resolution \\
P6 & 58 & Female & D3 & Fresh & Resolution \\
P7 & 67 & Male & D1 & Fresh & Resolution \\
P8 & 31 & Female & D3 & Frozen & Resolution \\
P9 & 35 & Female & D3 & Fresh & Resolution \\
P10 & 81 & Male & D3 & Fresh & Resolution \\
P11 & 80 & Female & D3 & Frozen & Resolution \\
P12 & 20 & Female & D3 & Frozen & Resolution \\
P13 & 57 & Female & D2 & Frozen & Resolution \\
P14 & 44 & Female & D3 & Frozen & Resolution \\
\hline
\end{tabular}

antimicrobial treatment and were refractive to standard therapy. One patient, P13, received FMT after one relapse only. She had previously suffered from three CDIs during the past 3 years, always coinciding with antibiotic treatment for other indications. The latest $C$. difficile infection started after a course of doxycycline and, due to the patient's history, FMT was considered as a suitable treatment and the patient was included in the study. The previously described clinical inclusion and exclusion criteria were followed [21]. The study was approved by the Ethics Committee of Hospital District of Helsinki and Uusimaa Finland (DnroHUS124/13/03/01/11). Patients were informed about the possible risks of FMT and they all provided informed consent.

\section{Donors and preparation of faecal transplants}

Three healthy Finnish females, aged 35-42 years and with a normal body mass index (average $23.5, \mathrm{SD}=2.1$ ) acted as universal faecal donors (D1-D3) and provided follow-up faecal samples (Fig. 1). The donors were screened as described previously [11]. In short, they did not have any gastrointestinal symptoms, had not taken antibiotics for the past 6 months, and were negative in C. difficile culture and toxin $\mathrm{A} / \mathrm{B}$ test. They were also negative for growth on selective culture for enteric bacterial pathogens and light microscopy on ova and parasites from faeces and as well tests for HBV, HCV, HIV-1, HIV-2 and Treponema pallidum from serum. Further tests included total blood count, C-reactive protein, creatinine and liver enzyme levels from blood.

The preparation of faecal suspensions for immediate use and for freeze-storing at $-80{ }^{\circ} \mathrm{C}$ was performed as described recently by using $30 \mathrm{~g}$ of faecal material [21]. The patients received an infusion of either fresh faeces 


Fecal transplantation
Fig. 1 Study design. Four to eight faecal samples were collected
from 14 patients and three donors over the 1-year study period, in
addition to two biopsy samples (from 10 patients only). F faecal
sample, $B$ biopsy sample

or previously frozen sample (Table 1 ). The frozen donor samples were stored at $-80{ }^{\circ} \mathrm{C}$ for a maximum of 4 months prior to transplantation.

\section{Faecal microbiota transplantation}

The patients were treated with vancomycin pre-FMT and the medication was discontinued in average 36 hours prior to treatment. Patients cleansed their bowels before FMT with polyethylene glycol [11, 21]. The faecal suspension was infused into the cecum. Patients were advised to contact the hospital if they had diarrhoea or other symptoms after FMT. Persisting diarrhoea with a positive $C$. difficile toxin stool test was considered as a treatment failure. The patients came for the second biopsy 1 month after the FMT (bowel not cleansed). In addition, the patients were paid a home visit 2 months after the transplantation and twice more during the 1year follow-up period to collect the stored faecal samples, which were kept in their home freezers at $-20{ }^{\circ} \mathrm{C}$ for 4 months.

\section{Samples and DNA extraction}

The baseline faecal samples were taken before the colonoscopy at home by the patients and brought to the clinic. The follow-up samples were frozen at $-20{ }^{\circ} \mathrm{C}$ immediately after defecation and stored in the patients home freezers for maximally 4 months until transfer to the laboratory for further analysis. Rectal biopsies were taken from the patients during the FMT (B0) and at 1 month post-FMT (B1) by proctoscopy (bowel not cleaned) and stored at $-80{ }^{\circ} \mathrm{C}$ until further processing. The patients and donors collected the baseline (F0) and follow-up (F1-F7) faecal samples (Fig. 1). Microbial DNA from patients $(n=131)$ and donors $(n=23)$ was extracted as described previously for biopsies and faecal samples according to current standard operation procedures, including a mechanical disruption of bacterial cells [24-26].

\section{Microbiota analysis}

Microbiota analysis was conducted with a benchmarked and validated phylogenetic microarray [27-30]. It covers the V1 and V6 hypervariable regions of the $16 \mathrm{~S}$ rRNA gene and targets over 1000 bacterial taxa detected in the human GI tract covering the major species. The raw signal intensities were normalised as described previously [29]. For faecal samples, the technical replicates with correlation over 0.96 were accepted for further analysis, and for biopsies and pre-FMT samples a slightly lower quality (over 0.95) was accepted due to the low microbial diversity in the samples. Pre-FMT samples from P13 did not meet these quality standards and were excluded from the analysis. The raw signal intensities were normalised as described previously and min-max algorithms were used for the between sample normalisation [29]. The probe-signal intensities were summarised to 130 genus-like and 22 phylum-like taxonomic groups.

The adherence of bacteria from donor faeces to 7-dayold Caco-2 cells was conducted as described previously [31] and detailed in Additional file 1. The amounts of adhered bacteria were analyzed with MiSeq sequencing of the 16S rRNA gene (detailed in Additional file 1).

\section{Statistical analysis}

All data analyses were carried out with logarithmtransformed data and performed using $\mathrm{R}$ (version 3.1.1). The similarity of the microbiota was determined using Spearman's rank correlation $(\rho)$. In the analysis comparing the similarity of patients' microbiota and their own donors, subject P13 was excluded due to both Crohn's disease and multiple antibiotic treatments during the follow-up period. Microbial diversity, a measure of microbial richness and evenness, was calculated by using the inverse Shannon diversity index. The variation in the data was visualised with principle component analysis (PCA). The differences between time points, similarity and diversity were tested with analysis of variance (ANOVA) with Tukey's honest significant differences post hoc analysis. The changes in the individual bacterial taxa between time points were assessed with a linear mixed model. All resulting $P$ values were adjusted for multiple comparisons using Benjamini-Hochberg false discovery rate and $P$ values below 0.05 were considered significant. The microbial profile separating the pre- and post-FMT groups was identified with redundancy analysis using bootstrap aggregation (baggedRDA) as described previously [32]. In determining the therapeutic core, a detection threshold of $<2.9 \log 10$ intensity, corresponding to approximately $0.13 \%$ relative abundance from the total bacterial amount, was used here.

\section{Results}

FMT resolved $\mathrm{rCDI}$ and restored healthy microbiota profiles in patients

The FMT treatment cleared rCDI from all patients. A single individual (P3) mistakenly restarted vancomycin after transplantation and developed CDI. She was 
treated successfully with a second FMT and remained asymptomatic throughout the follow-up period (for detailed analysis see Additional file 1: Figure S2).

The donors' microbiota was typical for healthy adults [28, 33-35], dominated by Firmicutes (85.0 \%), Actinobacteria ( $8.5 \%$ ) and Bacteroidetes (5.3 \%) (Fig. 2a) and showed significant individual-specific profiles. In contrast to the healthy donors, the patients' microbiota preFMT was extremely different. At the highest taxonomical level, 14 out of the 23 detected phylum-like taxa differed significantly between the donors and pre-FMT patients $(P<0.05$, Fig. 2a). The low levels of Clostridia and high levels of Bacilli and Proteobacteria contributed to the majority of the detected differences. When determining the genus-like taxa separating the pre-FMT patients and donors, we found 69 taxa to be significantly differently abundant in these two groups (Additional file 1: Table S2). Among them, there were 15 genus-like taxa that were increased in abundance by over 10 -fold, including bacteria related to Coprococcus eutactus (fold change $(\mathrm{FC})=24.71, P<0.05)$, Ruminococcus obeum (FC $=23.05, P<0.05)$ and Subdoligranulum variable $(\mathrm{FC}=$ 22.21, $P<0.05)$. Additionally, two genus-level taxa decreased drastically in abundance after FMT, i.e. bacteria related to Lactobacillus plantarum $(\mathrm{FC}=$ $-24.18, P<0.05)$ and Veillonella $(\mathrm{FC}=-40.64, P<0.05)$.

There was a dramatic difference in both diversity and microbiota composition after the FMT-treatment. The patients' microbial diversity increased significantly as early as 3 days post-FMT to resemble the donors and remained in this range for up to 1 year (Fig. 2c). A similar trend was observed with the microbial composition,

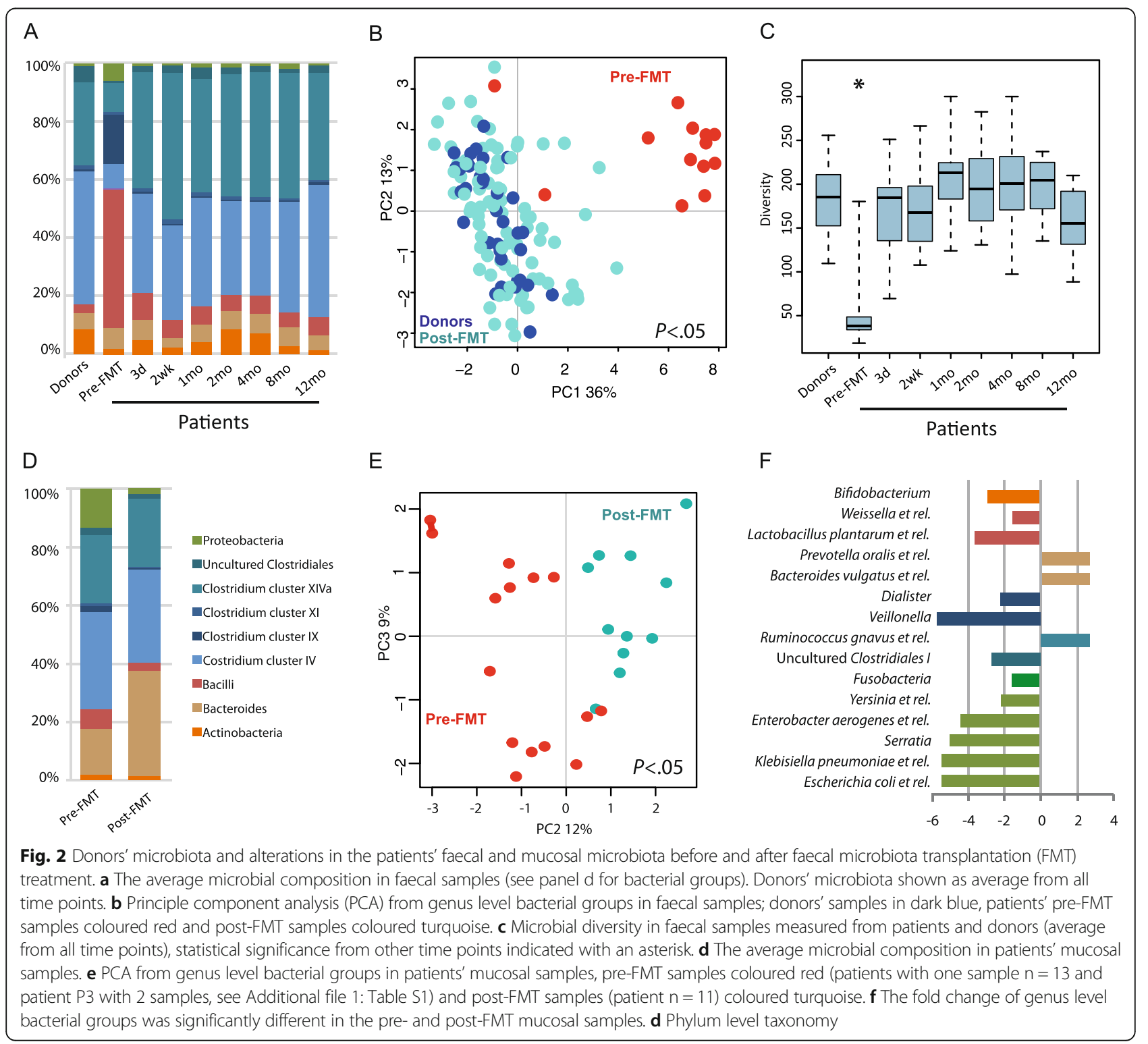


where patients' microbiota post-FMT resembled that of the donors throughout the follow-up period (Fig. 2a). This compositional shift is also seen from the unsupervised PCA plot, where $36 \%$ of the microbial variation was introduced by the treatment (Fig. 2b). There was no significant difference between any of the follow-up samples or healthy controls in the PCA.

\section{FMT-induced microbiota changes in the mucosal surface}

Microbiota changes in the intestinal mucosa of CDI patients have not been previously addressed. Interestingly, the changes of microbial profiles from rectal biopsies before and after FMT-treatment were different than what was observed in the faecal samples. The pre-treatment mucosal sample was significantly enriched with members of Clostridium cluster IX, Proteobacteria, Bacilli and uncultured Clostridiales, which were reduced postFMT, whereas Bacteroidetes were increased after treatment (all, $P<0.05$, Fig. 2 d). Furthermore, when analysing the effect of FMT on the genus-level taxa, there was a significant difference between pre- and post-treatment groups, which were separated in PCA $(P<0.05$, Fig. 2e). The separation was caused by 15 genus-level taxa (Fig. 2f). The largest difference was introduced by the 2.7-fold increase of the members of Bacteroidetes phylum, including Bacteroides vulgatus- and Prevotella oralis-related taxa after FMT, whereas members of the Proteobacteria phylum were decreased on average by 4.6-fold and bacteria related to Clostridium cluster IX, such as Veillonella spp., decreased. A baggedRDA analysis further supported the observed differences in the mucosal microbiota before and after FMT and confirmed that Proteobacterial and Clostridial species are decreased and the Bacteroidetes species are enriched after the FMT (Additional file 1: Figure S3). Surprisingly, FMT did not increase microbial diversity in the mucosa (Additional file 1: Table S3).

To analyse the adherence of donor faecal bacteria to intestinal epithelium in vitro we studied the most frequently used donor D3 and allowed the faecal sample to bind to the Caco-2 cell culture. The attached bacteria were analysed using 16S rRNA sequencing. Previously, high-throughput sequencing and the microarray platform used in this study have been shown to produce comparable data, particularly at high taxonomic level [30] and, therefore, we considered that it is adequate to analyse the Caco-2 adherent phyla with MiSeq sequencing. Interestingly, the in vitro result replicated our in vivo findings of increased levels of Bacteroidetes in the mucosa by showing a drastic decrease of the Firmicutes/ Bacteroidetes ratio from 31.31 to 7.45 in the faecal and Caco-2 adhered samples, respectively. This further suggests that specific bacteria from the faecal material are selected to the mucosal compartment.
Donor-specific microbiota established in the patients and retained for up to 1 year post-FMT

One of the main aims of this work was to evaluate the long-term persistence of the transplanted microbiota. To address this, we calculated the Spearman correlations measuring microbial similarity between three groups, namely the similarity between the donated sample and its recipient, the donors within-subject similarity against the donated sample over time, and similarity between the patient and other donors of the study. The high similarity between patients' and their donated sample throughout the follow-up period was striking (Fig. 3a). As early as 3-days after the treatment there was a $95.0 \%$ similarity between the patients' microbiota and the donated faeces, compared to the $81.8 \%$ similarity pre-FMT $(P<0.05)$. Importantly, similarity to the other donors was significantly lower than to the own donors $(P<0.05)$ and, furthermore, this similarity was retained throughout the study period (Fig. 3a). In addition, the overall similarity between the patient-donor pairs (average $95.3 \%$ ) was found to be remarkably higher than what is generally observed for unrelated individuals determined using the same analysis pipeline (average $77.4 \%, P<0.05$ ) $[25$, $28,34-36]$. Analysis of the microbiota stability at individual level showed that it had high resilience after FMT and, in three out four patients receiving antibiotics during the follow-up period, the microbiota was able to recover from the occasional antibiotic treatment for other indications (Additional file 1: Figure S4). As an exception, P13, who has Crohn's disease and received three courses of antibiotics, both of which are known to affect microbiota, had reduced stability (Additional file 1: Figure S4) and was therefore excluded from the cohort stability analysis.

Due to the high similarity between the donors and their patients, we investigated the possibility of microbial signatures in the patients that would be specific to their own donor. Using baggedRDA, we found that the patients could be separated according to their donor and observed 24 genus-like taxa to cause this separation (Fig. 3b). For example, bacteria related to Faecalibacterium prausnitzii, Ruminococcus lactaris and Collinsella were increased in the patients from D3. These signatures remained throughout the follow-up period.

\section{Commonly acquired bacterial taxa}

The universal donor approach of this study allowed identification of similarities introduced by the FMT. More specifically, we were able to identify genus-like bacterial taxa that were absent in the patients prior to treatment but introduced to the patients post-FMT (Fig. 4a). Each donor and their patients were first compared separately to achieve the donor-specific transplanted core microbiota. We then compared the three 

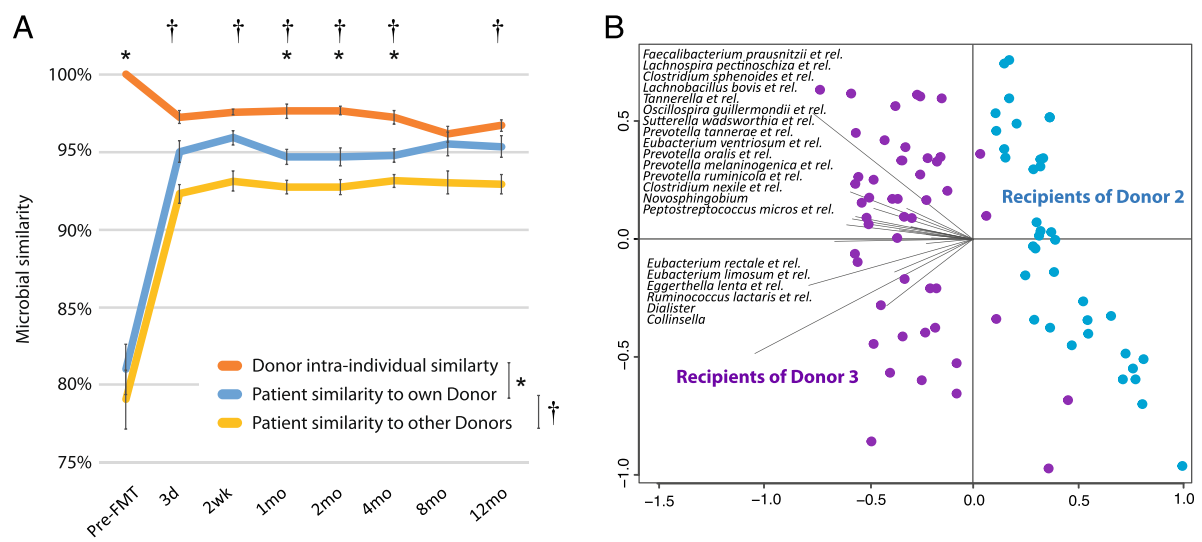

Fig. 3 Microbiota stability and donor specific microbiota signatures. a Similarity of the patients microbiota to their own donors' microbiota is significantly higher than the similarity to the other donor. Statistical significance between the groups are indicated with an asterisk (patient similarity to own donor vs. donor intra-individual similarity) and cross variation (patient similarity to own donor vs. patient similarity to other donors) is shown with standard error of mean (SEM). $\mathbf{b}$ Patient faecal samples present donor-specific microbial signatures in BaggedRDA analysis

donor-specific cores (Fig. 4b) and found that 24 genuslike taxa from four phylum-like groups were absent in patients prior to FMT and introduced by the treatment to at least two out of the three donors (Fig. 4b). Fifteen of these taxa were present in all patients after treatment. The commonly acquired bacteria included some wellstudied butyrate producers such as Eubacterium hallii and Roseburia intestinalis.

\section{Discussion}

Our study addressed the microbiological mechanisms underlying the FMT treatment for rCDI. We showed, for the first time, that FMT has long-term effects on the microbiota and offers a means to modify it relatively permanently. The rapid changes induced by FMT explain the prompt and high clinical efficacy - it drastically altered the patients' intestinal microbiota by restoring the anaerobic community. Patients' faecal microbiota prior to FMT was dominated with facultative anaerobic bacteria such as Bacilli and Proteobacteria, which are known for their proinflammatory properties [37]. PostFMT, their microbiota composition resembled that of the donors as early as 3 days following transplantation, containing bacteria typical for a healthy microbiota such as strict anaerobes from the Clostridium clusters IV and XIVa. These observed changes confirmed previous

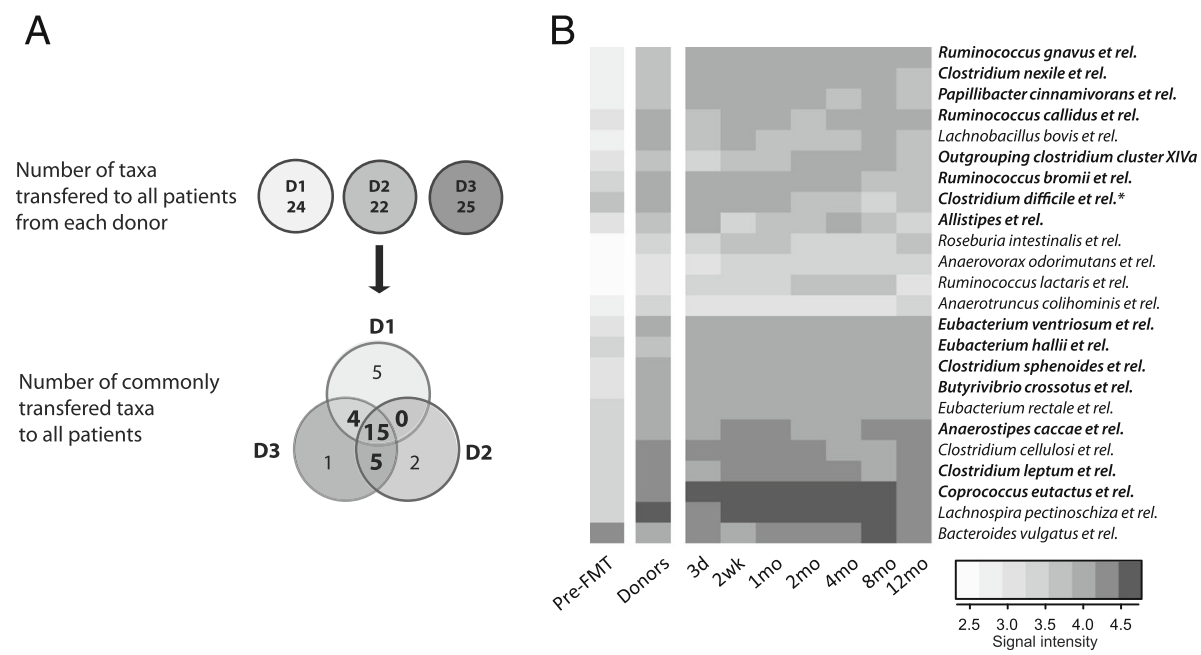

Fig. 4 The commonly acquired bacteria after faecal microbiota transplantation (FMT). a Flowchart showing how the commonly acquired bacteria were identified. $\mathbf{b}$ Heatmap showing the bacterial taxa, abundance and the stability of the therapeutic core. The bacterial groups shown with bold text were increased in all patients and the others were increased in patients from two out of three donors. ${ }^{*}$ The bacteria belonging to $C$. difficile group include eight commensal species and uncultured representatives (see Additional file 1), which produced the detected signal. C. difficile per se was absent from all donors and patients post-FMT 
findings $[14,38]$ and, importantly, we were able to show that these modifications persisted long-term. We also addressed the effects induced by FMT on the rectal mucosa, which have not been studied previously. Furthermore, our universal donor approach allowed the identification of commonly acquired bacterial taxa, potentially underlying the treatment efficacy.

Antibiotics suppress anaerobic commensals and induce profound changes to microbiota, resulting in loss of colonisation resistance $[39,40]$. We observed a similar effect in patient P3, who mistakenly took vancomycin after the first FMT. The transplanted microbiota was unable to engraft and there was no change in the microbial composition before the second FMT treatment. We also showed that the patients' microbiota composition pre-FMT represents the effects of multiple antibiotic treatments, including low diversity and depletion of anaerobes. The FMTtreatment restored these levels very rapidly.

The novel mucosal microbiota findings showed that similar to the faecal microbiota, FMT restored the anaerobic bacterial community due to the increase of Bacteroidetes. The faecal and mucosal tissues are distinct communities and have specific microbial compositions $[41,42]$. Therefore, it was not surprising that a subpopulation of the transplanted microbiota was selected to the mucosal compartment. Further, our in vitro experiment showed that the epithelium-adherent fraction of faecal microbiota was enriched in Bacteroidetes. This group is abundant in the healthy intestinal mucosa and is known to enforce epithelial integrity [43] and maintains immunological homeostasis $[44,45]$. Thus, it can be hypothesised that the increase of Bacteroidetes in the mucosa was part of the efficacy of the FMT treatment.

One of the main findings of this study was the high similarity in the microbiota profiles between patients and their own donors that lasted throughout the 1-year follow-up. This was not altered even by antimicrobial treatments taken by some patients during the follow-up period. The microbial stability was effected by the antibiotics, but it recovered to its original composition, in line with recent observations with healthy subjects [40]. Regardless of the antibiotics, we were able to identify specific donor-derived bacterial signatures, which persisted throughout the follow-up. This surprisingly high similarity between the donor-patient pair led us to speculate that there is no major selection pressure from the host to alter the transplanted microbial composition. The hypothesis could be that the transplant provides a functional microbial ecosystem, which outweighs the individual-based bacterial selection.

Previously, three FMT-trials have addressed the engraftment of donors' microbiota in patients, with shorter 4- to 6-month follow-up periods and less detailed microbial analysis [17, 38]. Our comprehensive investigation extends the previous preliminary observations on the establishment of donors' microbiota postFMT; both the high patient-donor similarity and the donor-specific bacterial signatures in patients indicate a long-term establishment of the donors' microbiota. This is in line with a recent metagenomics study that revealed colonisation of donor bacteria at strain level persisting for 3 months after FMT treatment [46]. Since one of the characteristics of a healthy microbiota is its resilience to change [35], it was unexpected that the donors' microbiota was so strongly established and maintained. Our hypothesis is that the depletion of the microbiota with broad-spectrum antibiotics and bowel cleansing creates an open ecological niche for the transplanted microbiota. This novel finding on the long-term stability is promising when considering other indications where changing the intestinal microbiota composition could be used as a potential treatment.

One of our main aims was to determine a group of bacteria necessary for the resolution rCDI. This was addressed by the universal study setup, where faecal preparations from three donors were used to treat several patients, allowing better evaluation of the commonly acquired bacteria, which were transferred to all patients. We identified 24 bacterial taxa that were absent in patients before the treatment and present afterwards. Thus, it would be plausible to hypothesise that such a specific subpopulation within the complex faecal microbiota could underlie the treatment efficacy of FMT for rCDI. This commonly acquired core identified in our study was taxonomically diverse and included bacterial genera from four major phyla. The therapeutic core determined in our study showed considerable overlap with healthassociated microbial cores determined in other studies [47], highlighting its potential in restoring health.

The impact of these 24 taxa to intestinal health potentially lies in their ecological functions and nutrient utilisation networks as well as immunomodulatory capacity. One of these genera, Bacteroides spp. has been previously found to increase significantly after FMT for rCDI and to have a key role in restoring the intestinal ecosystem [14]. Our findings on the increase of Bacteroides spp. in the mucosa also underline their importance in maintaining intestinal homeostasis. There is evidence that the human commensal B. fragilis fortifies epithelial integrity [43] and, more recently, the bacterium was shown to interact with intestinal mucosa to suppress inflammation [48]. Furthermore, mice studies have shown that the Bacteroidetes taxa are required in successful colonisation of a healthassociated Faecalibacterium prauznitzii [49].

The majority $(22 / 24)$ of the commonly transplanted bacterial taxa belonged to three Clostridium clusters (Firmicutes). The Clostridium taxa of the therapeutic core have been shown to play key roles in the nutrient 
utilisation networks and, therefore, can be considered to be essential for the general restoration of the complex ecosystem [50-52]. For example, the therapeutic core bacteria Eubacterium, Coprococcus, Anaerostipes and Ruminococcus spp. are known to participate in bacterial cross-feeding pathways that are responsible for the production of short-chain fatty acids (SCFA) - the major microbial metabolites from carbohydrate fermentation [50]. Concurrently, with the appearance of the therapeutic core taxa, we also observed a more than 20-fold increase in Ruminococcus obeum and Subdoligranulum variable, both of which are major SCFA-producing bacteria in the gut [50]. SCFAs promote intestinal homeostasis by both strengthening epithelial cell layer integrity and stimulating regulatory T cells [53]. Recently, Atarashi et al. [54] treated inflammatory colitis in a mouse model with a combination of 17 clostridial strains, which affected SCFA and regulatory $\mathrm{T}$ cell levels.

In summary, the therapeutic core seems to consist of intestinal bacteria that are able to regenerate key interactionnetworks within the microbiota and consequently restore the complex intestinal ecosystem that carries out essential functions for the host and provides colonisation resistance against pathogens, especially $C$. difficile. Therefore, isolation and characterisation of these commensal bacteria would be of high importance when developing microbiotabased therapies for rCDI. We consider that there are multiple alternatives to combine intestinal bacterial strains as an effective bacteriotherapy mixture.

\section{Conclusions}

The microbiota changes both in faeces and mucosa explain the rapid clinical recovery of all patients and the superior long-term efficacy over previous antibiotic treatments. Our results indicate that a specific combination of bacterial taxa seems to underlie the treatment efficacy of FMT for rCDI. This is the first study to show that subject's microbiota could be modified in the longterm to resemble that of the donor. Currently, FMT treatment is considered for several other indications than just the treatment of rCDI. Therefore, our findings give insights into the possibilities of reshaping patients' microbiota relatively permanently.

\section{Additional file}

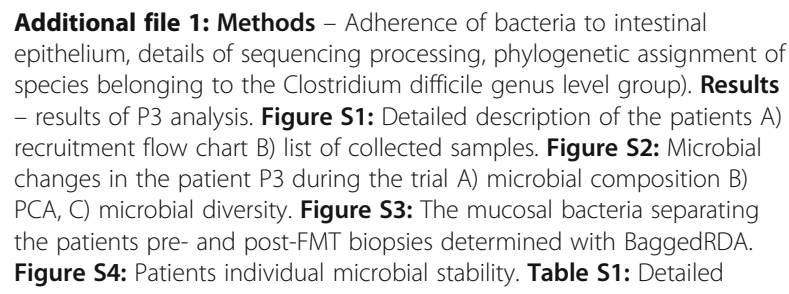

patient demographics. Table S2: Significant differences between the preand post-FMT samples on genus-like taxonomical level. Table S3: Microbial diversity of the mucosal samples. (PDF $2230 \mathrm{~kb}$ )

\section{Abbreviations}

BaggedRDA: Bootstrap aggregated redundancy analysis; FMT: Faecal microbiota transplant; PCA: Principle component analysis; rCDI: Recurrent Clostridium difficile infection; SCFA: Short-chained fatty acids

\section{Acknowledgments}

We would like to warmly thank Lars Paulin from the Institute of Biotechnology, University of Helsinki, for his assistance with the MiSeq analysis, Dr. Jarkko Salojärvi for the development of the BaggedRDA method, and the HITChip team for their brilliant technical assistance. We would also like thank the funding agencies Academy of Finland (258439 and 283088) and the Finnish foundation for Gastroenterological Research. Finally, we would like to thank the donors and patients for their participation in the study.

\section{Funding}

Academy of Finland grants 258439 and 283088 and the Finnish Foundation for Gastroenterological Research.

\section{Author contributions}

Study concept and design: EM, WdV, PA, RS. Acquisition of data: JJ, EM, HJ, $J H, P A, R S$. Analysis of data: JJ, HJ, JH, RS. Interpretation of data: JJ, EM, WdV, PA, RS. Drafting of the manuscript: JJ, RS. Statistical analysis: JJ. Obtained funding: RS. Critical revision of the manuscript for important intellectual content: all authors. All authors read and approved the final manuscript.

\section{Competing interests}

The authors declare that they have no competing interests.

\section{Author details}

${ }^{1}$ Immunobiology Research Program and Department of Bacteriology and Immunology, Faculty of Medicine, University of Helsinki, Helsinki, Finland. ${ }^{2}$ Department of Infectious Disease, Helsinki University Central Hospital, Helsinki, Finland. ${ }^{3}$ Laboratory of Microbiology, Wageningen University, Wageningen, The Netherlands. ${ }^{4}$ Department of Gastroenterology, Helsinki University Central Hospital, Helsinki, Finland. ${ }^{5}$ PO Box 21, Haartmaninkatu 3, 00290 Helsinki, Finland.

Received: 25 May 2016 Accepted: 16 September 2016

Published online: 11 October 2016

\section{References}

1. Kelly CP, LaMont JT. Clostridium difficile - More difficult than ever. New Engl J Med. 2008;359(18):1932-40.

2. Louie TJ, Miller MA, Mullane KM, Weiss K, Lentnek A, Golan Y, Gorbach S, Sears P, Shue YK, OPT-80-003 Clinical Study Group. Fidaxomicin versus vancomycin for Clostridium difficile infection. New Engl J Med. 2011;364(5): 422-31.

3. Honda H, Dubberke ER. The changing epidemiology of Clostridium difficile infection. Curr Opin Gastroenterol. 2014;30(1):54-62.

4. Rupnik M, Wilcox MH, Gerding DN. Clostridium difficile infection: new developments in epidemiology and pathogenesis. Nat Rev Microbiol. 2009; 7(7):526-36.

5. Surawicz CM, Brandt LJ, Binion DG, Ananthakrishnan AN, Curry SR, Gilligan PH, McFarland LV, Mellow M, Zuckerbraun BS. Guidelines for diagnosis, treatment, and prevention of Clostridium difficile infections. Am J Gastroenterol. 2013;108(4):478-98. quiz 499.

6. Mattila E, Arkkila P, Mattila PS, Tarkka E, Tissari P, Anttila VJ. Rifaximin in the treatment of recurrent Clostridium difficile infection. Aliment Pharmacol Ther. 2013;37(1):122-8.

7. Garey KW, Ghantoji SS, Shah DN, Habib M, Arora V, Jiang ZD, DuPont HL. A randomized, double-blind, placebo-controlled pilot study to assess the ability of rifaximin to prevent recurrent diarrhoea in patients with Clostridium difficile infection. J Antimicrob Chemother. 2011;66(12): 2850-5. 
8. Borody TJ, Khoruts A. Fecal microbiota transplantation and emerging applications. Nat Rev Gastro Hepat. 2012;9(2):88-96.

9. Tenover FC, Tickler IA, Persing DH. Antimicrobial-resistant strains of Clostridium difficile from North America. Antimicrob Agents Chemother. 2012;56(6):2929-32.

10. Brandt $\amalg$, Aroniadis OC, Mellow M, Kanatzar A, Kelly C, Park T, Stollman N, Rohlke F, Surawicz C. Long-term follow-up of colonoscopic fecal microbiota transplant for recurrent Clostridium difficile infection. Am J Gastroenterol. 2012;107(7):1079-87.

11. Mattila E, Uusitalo-Seppala R, Wuorela M, Lehtola L, Nurmi H, Ristikankare M, Moilanen V, Salminen K, Seppala M, Mattila PS, et al. Fecal transplantation, through colonoscopy, is effective therapy for recurrent Clostridium difficile infection. Gastroenterology. 2012;142(3):490-6.

12. van Nood E, Vrieze A, Nieuwdorp M, Fuentes S, Zoetendal EG, de Vos WM Visser CE, Kuijper EJ, Bartelsman JF, Tijssen JG, et al. Duodenal infusion of donor feces for recurrent Clostridium difficile. N Engl J Med. 2013;368(5): 407-15

13. Kassam Z, Lee $\mathrm{CH}$, Yuan $Y$, Hunt RH. Fecal microbiota transplantation for Clostridium difficile infection: systematic review and meta-analysis. Am J Gastroenterol. 2013;108(4):500-8.

14. Fuentes S, van Nood E, Tims S, Heikamp-de Jong I, ter Braak CJ, Keller JJ, Zoetendal EG, de Vos WM. Reset of a critically disturbed microbial ecosystem: faecal transplant in recurrent Clostridium difficile infection. ISME J. 2014;8(8):1621-33.

15. Brace C, Gloor GB, Ropeleski M, Allen-Vercoe E, Petrof EO. Microbial composition analysis of Clostridium difficile infections in an ulcerative colitis patient treated with multiple fecal microbiota transplantations. J Crohns Colitis. 2014;8(9):1133-7.

16. Shankar V, Hamilton MJ, Khoruts A, Kilburn A, Unno T, Paliy O, Sadowsky MJ. Species and genus level resolution analysis of gut microbiota in Clostridium difficile patients following fecal microbiota transplantation. Microbiome. 2014;2:13.

17. Weingarden A, Gonzalez A, Vazquez-Baeza Y, Weiss S, Humphry G, BergLyons D, Knights D, Unno T, Bobr A, Kang J, et al. Dynamic changes in short- and long-term bacterial composition following fecal microbiota transplantation for recurrent Clostridium difficile infection. Microbiome. 2015;3:10.

18. Lee $\mathrm{CH}$, Belanger JE, Kassam Z, Smieja M, Higgins D, Broukhanski G, Kim PT. The outcome and long-term follow-up of 94 patients with recurrent and refractory Clostridium difficile infection using single to multiple fecal microbiota transplantation via retention enema. Eur J Clin Microbiol. 2014; 33(8):1425-8.

19. Weingarden AR, Dosa PI, DeWinter E, Steer CJ, Shaughnessy MK, Johnson JR, Khoruts A, Sadowsky MJ. Changes in colonic bile acid composition following fecal microbiota transplantation are sufficient to control Clostridium difficile germination and growth. PLoS One. 2016;11(1), e0147210.

20. Buffie CG, Bucci V, Stein RR, McKenney PT, Ling L, Gobourne A, No D, Liu H, Kinnebrew $M$, Viale $A$, et al. Precision microbiome reconstitution restores bile acid mediated resistance to Clostridium difficile. Nature. 2015;517(7533): 205-8.

21. Satokari R, Mattila E, Kainulainen V, Arkkila PE. Simple faecal preparation and efficacy of frozen inoculum in faecal microbiota transplantation for recurrent Clostridium difficile infection-an observational cohort study. Aliment Pharmacol Ther. 2015;41(1):46-53.

22. Lawley TD, Clare S, Walker AW, Stares MD, Connor TR, Raisen C, Goulding D, Rad R, Schreiber F, Brandt C, et al. Targeted restoration of the intestinal microbiota with a simple, defined bacteriotherapy resolves relapsing Clostridium difficile disease in mice. PLoS Pathog. 2012;8(10), e1002995.

23. Petrof EO, Gloor GB, Vanner SJ, Weese SJ, Carter D, Daigneault MC, Brown EM, Schroeter K, Allen-Vercoe E. Stool substitute transplant therapy for the eradication of Clostridium difficile infection: 'RePOOPulating' the gut. Microbiome. 2013;1(1):3.

24. Kalliomaki M, Satokari R, Lahteenoja H, Vahamiko S, Gronlund J, Routi T, Salminen S. Expression of microbiota, Toll-like receptors, and their regulators in the small intestinal mucosa in celiac disease. J Pediatr Gastroenterol Nutr. 2012;54(6):727-32.

25. Salonen A, Nikkilä J, Jalanka-Tuovinen J, Immonen O, Rajilic-Stojanovic M, Kekkonen RA, Palva A, de Vos WM. Comparative analysis of fecal DNA extraction methods with phylogenetic microarray: effective recovery of bacterial and archaeal DNA using mechanical cell lysis. J Microbiol Methods. 2010;81(2):127-34
26. Dore J, Ehrlich SD, Levenez F, Pelletier E, Alberti A, Bertrand L, Bork P, Costea PI, Sunagawa S, Guarner F, Manichanh C, Santiago A, Zhao L, Shen J, Zhang C, Versalovic J, Luna RA, Petrosino J, Yang H, Li S, Wang J, Allen-Vercoe E, Gloor G, Singh B; IHMS Consortium. IHMS_SOP 06V1: Standard operating procedure for fecal samples DNA extraction, Protocol Q. Int Hum Microbiome Standards. 2015. www.microbiome-standards.org/index.php?id=253. Accessed 24 May 2016.

27. O'Keefe SJ, Li JV, Lahti L, Ou J, Carbonero F, Mohammed K, Posma JM, Kinross J, Wahl E, Ruder $E$, et al. Fat, fibre and cancer risk in African Americans and rural Africans. Nat Commun. 2015;6:6342

28. Rajilic-Stojanovic M, Heilig HG, Molenaar D, Kajander K, Surakka A, Smidt H, de Vos WM. Development and application of the human intestinal tract chip, a phylogenetic microarray: analysis of universally conserved phylotypes in the abundant microbiota of young and elderly adults. Environ Microbiol. 2009;11(7):1736-51.

29. Lahti L, Salojarvi J, Salonen A, Scheffer M, de Vos WM. Tipping elements in the human intestinal ecosystem. Nat Commun. 2014;5:4344.

30. Claesson MJ, O'Sullivan O, Wang Q, Nikkilä J, Marchesi JR, Smidt H, de Vos WM, Ross RP, OToole PW. Comparative analysis of pyrosequencing and a phylogenetic microarray for exploring microbial community structures in the human distal intestine. PLoS One. 2009;4(8), e6669.

31. Kainulainen V, Reunanen J, Hiippala K, Guglielmetti S, Vesterlund S, Palva A, Satokari R. BopA does not have a major role in the adhesion of Bifidobacterium bifidum to intestinal epithelial cells, extracellular matrix proteins, and mucus. Appl Environ Microbiol. 2013;79(22):6989-97.

32. Jalanka-Tuovinen J, Salojarvi J, Salonen A, Immonen O, Garsed K, Kelly FM, Zaitoun A, Palva A, Spiller RC, de Vos WM. Faecal microbiota composition and host-microbe cross-talk following gastroenteritis and in postinfectious irritable bowel syndrome. Gut. 2014;63(11):1737-45.

33. Qin J, Li R, Raes J, Arumugam M, Burgdorf KS, Manichanh C, Nielsen T, Pons $\mathrm{N}$, Levenez F, Yamada T, et al. A human gut microbial gene catalogue established by metagenomic sequencing. Nature. 2010;464(7285):59-65.

34. Jalanka-Tuovinen J, Salonen A, Nikkilä J, Immonen O, Kekkonen R, Lahti L, Palva A, de Vos WM. Intestinal microbiota in healthy adults: temporal analysis reveals individual and common core and relation to intestinal symptoms. PLoS One. 2011;6(7), e23035

35. Rajilic-Stojanovic M, Heilig HG, Tims S, Zoetendal EG, de Vos WM. Long-term monitoring of the human intestinal microbiota composition. Environ Microbiol. 2013;15(4):1146-59.

36. Tims S, Derom C, Jonkers DM, Vlietinck R, Saris WH, Kleerebezem M, de Vos WM, Zoetendal EG. Microbiota conservation and BMI signatures in adult monozygotic twins. ISME J. 2013;7(4):707-17.

37. Round $J$, Mazmanian SK. The gut microbiota shapes intestinal immune responses during health and disease. Nat Rev Immunol. 2009;9(5):313-23.

38. Hamilton MJ, Weingarden AR, Unno T, Khoruts A, Sadowsky MJ. Highthroughput DNA sequence analysis reveals stable engraftment of gut microbiota following transplantation of previously frozen fecal bacteria. Gut Microbes. 2013;4(2):125-35.

39. Dethlefsen L, Huse S, Sogin ML, Relman DA. The pervasive effects of an antibiotic on the human gut microbiota, as revealed by deep $16 \mathrm{~S}$ rRNA sequencing. PLoS Biol. 2008;6(11), e280

40. Rashid MU, Zaura E, Buijs MJ, Keijser BJF, Crielaard W, Nord CE, Weintraub A. Determining the long-term effect of antibiotic administration on the human normal intestinal microbiota using culture and pyrosequencing methods. Clin Infect Dis. 2015;60:577-84.

41. Zoetendal EG, von Wright A, Vilpponen-Salmela T, Ben-Amor K, Akkermans $A D$, de Vos WM. Mucosa-associated bacteria in the human gastrointestinal tract are uniformly distributed along the colon and differ from the community recovered from feces. Appl Environ Microbiol. 2002;68(7): 3401-7.

42. Satokari R, Fuentes S, Mattila E, Jalanka J, de Vos WM, Arkkila P. Fecal transplantation treatment of antibiotic-induced, noninfectious colitis and long-term microbiota follow-up. Case Rep Med. 2014;2014:913867.

43. Reunanen J, Kainulainen V, Huuskonen L, Ottman N, Belzer C, Huhtinen H, de Vos WM, Satokari R. Akkermansia muciniphila adheres to enterocytes and strengthens the integrity of the epithelial cell layer. Appl Environ Microbiol. 2015:81(11):3655-62.

44. Johnson JL, Jones MB, Cobb BA. Polysaccharide A from the capsule of Bacteroides fragilis induces clonal CD4+ T cell expansion. J Biol Chem. 2015 290(8):5007-14.

45. Wang Y, Telesford KM, Ochoa-Reparaz J, Haque-Begum S, Christy M, Kasper EJ, Wang L, Wu Y, Robson SC, Kasper DL, et al. An intestinal commensal 
symbiosis factor controls neuroinflammation via TLR2-mediated CD39 signalling. Nat Commun. 2014;5:4432.

46. Li SS, Zhu A, Benes V, Costea PI, Hercog R, Hildebrand F, Huerta-Cepas J, Nieuwdorp M, Salojarvi J, Voigt AY, et al. Durable coexistence of donor and recipient strains after fecal microbiota transplantation. Science. 2016; 352(6285):586-9.

47. Cheng J, Ringel-Kulka T, Heikamp-de Jong I, Ringel Y, Carroll I, de Vos WM, Salojarvi J, Satokari R. Discordant temporal development of bacterial phyla and the emergence of core in the fecal microbiota of young children. ISME J. 2016;10(4):1002-14.

48 Chu H, Khosravi A, Kusumawardhani IP, Kwon AH, Vasconcelos AC, Cunha LD, Mayer AE, Shen Y, Wu WL, Kambal A, et al. Gene-microbiota interactions contribute to the pathogenesis of inflammatory bowel disease. Science. 2016;352(6289):1116-20.

49 Wrzosek L, Miquel S, Noordine ML, Bouet S, Chevalier-Curt MJ, Robert V, Philippe C, Bridonneau C, Cherbuy C, Robbe-Masselot C, et al. Bacteroides thetaiotaomicron and Faecalibacterium prausnitzii influence the production of mucus glycans and the development of goblet cells in the colonic epithelium of a gnotobiotic model rodent. BMC Biol. 2013;11:61.

50 Louis P, Hold GL, Flint HJ. The gut microbiota, bacterial metabolites and colorectal cancer. Nat Rev Microbiol. 2014;12(10):661-72.

51 Duncan SH, Louis P, Flint HJ. Lactate-utilizing bacteria, isolated from human feces, that produce butyrate as a major fermentation product. Appl Environ Microbiol. 2004;70(10):5810-7.

52 Watanabe $Y$, Nagai F, Morotomi M. Characterization of Phascolarctobacterium succinatutens sp nov., an asaccharolytic, succinateutilizing bacterium isolated from human feces. Appl Environ Microbiol. 2012;78(2):511-8

53 Lopez CA, Kingsbury DD, Velazquez EM, Baumler AJ. Collateral damage: microbiota-derived metabolites and immune function in the antibiotic era. Cell Host Microbe. 2014;16(2):156-63.

54 Atarashi K, Tanoue T, Oshima K, Suda W, Nagano Y, Nishikawa H, Fukuda S, Saito T, Narushima S, Hase K, et al. Treg induction by a rationally selected mixture of Clostridia strains from the human microbiota. Nature. 2013; 500(7461):232-6.

\section{Submit your next manuscript to BioMed Central and we will help you at every step:}

- We accept pre-submission inquiries

- Our selector tool helps you to find the most relevant journal

- We provide round the clock customer support

- Convenient online submission

- Thorough peer review

- Inclusion in PubMed and all major indexing services

- Maximum visibility for your research

Submit your manuscript at www.biomedcentral.com/submit 